\title{
Low-dimensional phase-locked states in the Zakharov equations
}

\author{
G. I. de Oliveira, L. P. L. de Oliveira, and F. B. Rizzato \\ Instituto de Física, Universidade Federal do Rio Grande do Sul, Caixa Postal 15051, \\ 91501-970 Porto Alegre, Rio Grande do Sul, Brazil
}

(Received 21 December 1995)

\begin{abstract}
In this paper we identify phase-locked states among the solutions of the Zakharov equations. Locked states appear as resonant island chains in the appropriate Poincare plots, with the relevant surface of section obtained by projecting out the full dynamical set on a subspace defined in terms of a pair of center-manifold variables. This pair allows an accurate canonical description of the system immediately after an inverse pitchfork bifurcation destabilizes an initial homogeneous steady state. If one is very close to the bifurcation point, nonlinear saturation of the initial instability is provided by quasistatic integrable ion-acoustic fluctuations, but as one proceeds away from that point, resonant nonintegrable ion-acoustic fluctuations become gradually more important; we show that the phase-locked states result from those resonant fluctuations. If one is not too far from the pitchfork bifurcation, locking is the stable asymptotic state of the interaction. As one moves farther away, locking exists only over long but finite amounts of time. In addition, the resonance separatrix appears to bring the first chaotic activity into the system. [S1063-651X(96)09109-X]
\end{abstract}

PACS number(s): 05.45.+b

\section{INTRODUCTION}

Langmuir turbulence has been one of the most studied problems in modern nonlinear plasma physics. During the past years a great deal of effort has been directed to its analysis, as well as to the analysis of related subjects as soliton dynamics, collapse, nucleation of cavitons, electromagnetic emission, and others [1-6].

There has been a growing tendency in looking at Langmuir turbulence as a result of chaos in nonlinear dynamical systems. The nonlinear system here is described by the $\mathrm{Za}$ kharov equations that couple the slowly varying amplitude of a high-frequency electric field, the Langmuir field, to slow density fluctuations, the ion-acoustic field.

Following this line of reasoning, all the tools and language so usual in the study of nonlinear dynamics have been used to search for signatures revealing chaotic activity, as well as transition to chaos, in both the dissipative and conservative versions of the turbulence [3-6]. In the presence of dissipation the system has been found to be accurately described by a reduced set of dissipative nonlinear equations [5]. On the other hand, under certain conditions the turbulence can be modeled in terms of a one-dimensional conservative nonlinear system. Indeed, it has been shown that if the system is placed in a moderately strong background magnetic field, electrostatic disturbances tend to align along the field - the transverse growth rate is much smaller than the longitudinal one - which otherwise does not exceedingly affect the qualitative aspect of the wave dynamics as long as the electron-cyclotron frequency is smaller than the electronplasma frequency; in that case the basic characteristics of the turbulence could be described in terms of a one-dimensional unmagnetized model [5]. As for the conservative character of the model the justification goes as follows. Dissipation is crucial if one studies large values of the wave vector where the resonant wave-particle interaction is relevant. On the other hand, the wave-particle interaction and dissipation are greatly reduced if the analysis does consider pump amplitudes just below the threshold for the inverse cascade that transport energy from low to large values of wave vectors.

Here we will be working with regimes dominated by modulational processes where pump amplitudes are below the threshold for the inverse cascade. Thus all the features we detect involve wave vectors somewhat trapped in a range of small magnitudes where dissipation is not as crucial $[3,4,6]$. In other words, we shall analyze the one-dimensional Zakharov equations without sources and dissipation.

As mentioned, our basic interest is to investigate the modulational behavior of the system immediately after an inverse pitchfork bifurcation rendering a homogeneous steady state unstable. We will see that by varying the appropriate parameters, the transition to chaos, if any, does take place close to that bifurcation. Besides, and precisely because one is close to a bifurcation, we shall see that the entire multidimensional system may be dealt with in a centermanifold-like fashion, displaying therefore characteristics of low-dimensional dynamics. In concrete terms, the type of transition we detect here involves phase-locked states among a few active modes. Locking does take place when a triplet formed by high-frequency modes and the corresponding enslaved, or quasistatic, ion-acoustic fluctuations starts to interact nonlinearly with ion-acoustic-free, or normal, modes.

From the above paragraph, it is thus seen that to observe locking one must be somewhat close to the pitchfork bifurcation; but the situation can be further detailed. We have found that if the parameters are such that one is sufficiently near the pitchfork point, locking is stable and represents asymptotic states of wave interaction. On the other hand, as one starts to move away from that point, locking turns out to be transient. In that case higher harmonics of the highfrequency field detunes the interaction after long but finite periods of time.

Technically, to describe the modulational instability we make use of a spectral analysis and write all the relevant 
dynamical variables as Fourier series in terms of a basic wave vector. As seen in the previous paragraphs, we shall analyze the dynamics just as it departs from its steady state. Therefore, we follow the usual procedure adopted in calculations involving transition to modulational instability [4] and choose a wave vector that varies around its critical value where the homogeneous equilibrium goes unstable via the pitchfork bifurcation. The basic wave vector introduced this way is an important parameter in determining all relevant frequencies of the system. Our approach renders the system spatially periodic since the basic wave vector creates a periodicity length. But from the above comments we can actually expect some characteristic wave vector in weakly unstable regimes, the wave vector being close to the critical. We mention that periodic solutions both in time and space are thought to be present in magnetospheric plasmas such as those found in a pulsar [7]. In fact, the observed periodic radiation pulses emitted by these objects are believed to be formed when the amplitude of homogeneous trains of electromagnetic radiation crosses the threshold and causes the train to become modulationally unstable: in this kind of environment, the dynamics is likely to be described by a nonlinear Schrödinger equation, which is a particular case of the equations analyzed here. In any case, apart from placing the basic wave vector near the instability threshold, no further assumptions are used here in the sense that all the subsequent dynamics, including harmonic generation, is self-consistently governed by the nonlinear interactions.

The paper is organized as follows. In Sec. II we write down the governing equations and derive a low-dimensional integrable description of the system: the initial inverse pitchfork bifurcation destabilizing a homogeneous steady state is pointed out and the integrable low-dimensional topology on the appropriate phase space is described: In Sec. III we pinpoint the dynamical variable responsible for the transition to chaos. In addition, signatures of the transition, appearing in the form of phase-locked states or nonlinear resonant islands, are identified. In Sec. IV a low-dimensional nonintegrable model comprising the basic features of the transition is constructed and compared with further simulations of the real system. In Sec. V we conclude the work.

\section{GOVERNING EQUATIONS AND A LOW-DIMENSIONAL INTEGRABLE MODEL}

The one-dimensional Zakharov equations governing the Langmuir turbulence can be written in the adimensional form [6]

$$
i \partial_{t} E+\partial_{x}^{2} E=n E
$$

and

$$
\partial_{t}^{2} n-\partial_{x}^{2} n=\partial_{x}^{2}|E|^{2} .
$$

$E(x, t)$ is the slowly modulated amplitude of the highfrequency electric field, the Langmuir field, and $n(x, t)$ is the low-frequency density fluctuation associated with the ionacoustic field.

Let us assume that the system is placed in a spatially periodic, one-dimensional box of length $L$. Then one can expand the fields $E(x, t)$ and $n(x, t)$ into Fourier series as

$$
E(x, t)=\sum_{m=-\infty}^{+\infty} \sqrt{\rho_{m}(k, t)} e^{i \phi_{m}(k, t)} e^{i m k x}
$$

and

$$
n(x, t)=\sum_{m=-\infty}^{+\infty} n_{m}(k, t) e^{i m k x}
$$

where the basic wave vector is defined in terms of $L$ as $k=2 \pi / L$ and the amplitudes $\rho_{m}(k, t)$ and phases $\phi_{m}(k, t)$ are conveniently introduced.

We now focus attention on initially homogeneous dipolar states whose fields are given by $E^{\text {dipolar }}(x, t=0)=\sqrt{\rho_{0}^{\text {dipolar }}} \equiv \sqrt{\rho_{*}} \quad$ and $\quad n^{\text {dipolar }}(x, t=0)=0$. The analysis is performed for a dipolar pump with $k=0$, but we expect that the results hold for nonvanishing values of the pump wave vector provided that the system be modulational but not parametrically unstable.

The stability of the dipolar state can be examined if it is perturbed with a small disturbance $\rho_{ \pm 1}, n_{ \pm 1} \ll \rho_{*}$. In the subsonic regime, one obtains for the corresponding growth rate $\Gamma$,

$$
\Gamma \sim k \sqrt{2 \rho_{*}-k^{2}},
$$

from which one sees that instability sets in only if $k^{2}<2 \rho_{*}$. It has been shown [6] that the destabilization is accompanied by an inverse pitchfork bifurcation where an unstable fixed point bifurcates into one stable and two new unstable points; we shall locate the fixed points later on. Now, it suffices to note that only if

$$
\rho_{*} \ll 1
$$

or if the above inequality is not true,

$$
\left|2 \rho_{*}-k^{2}\right| \ll 1,
$$

the dynamics is really subsonic in the sense that the growth rate is much smaller than $|k|$, the basic frequency of ionacoustic normal modes. One can better visualize the region defined by the relations (6) and (7) on a $\rho_{*} \times k$ parametric plane; this is done in Fig. 1. Above curve $k_{u}\left(\rho_{*}\right)$, which is analytically represented by $k_{u}=\sqrt{2 \rho_{*}}$, the homogeneous state is stable and below the curve it becomes unstable; condition (7), in turn, defines a narrow band very close to curve $k_{u}\left(\rho_{*}\right)$. Below curve $k_{u} / 2$, not only is the mode with wave vector $|k|$ unstable but also the mode with a higher harmonic wave vector $2|k|$; further reduction of $|k|$ gradually destabilizes more and more higher harmonics. All these facts lead us to think that if $k$ and $\rho_{*}$ are such that either condition (6) along with $k>k_{u} / 2$ or condition (7) is satisfied, the dynamics may be well described only in terms of the three linearly unstable high-frequency modes

$$
E_{\text {trunc }}(x, t)=\sum_{m=-1,0,+1} \sqrt{\rho_{m}(t)} e^{i \phi_{m}(t)} e^{i m k x},
$$

and enslaved ion-acoustic fluctuations obtainable from the quasistatic equation 


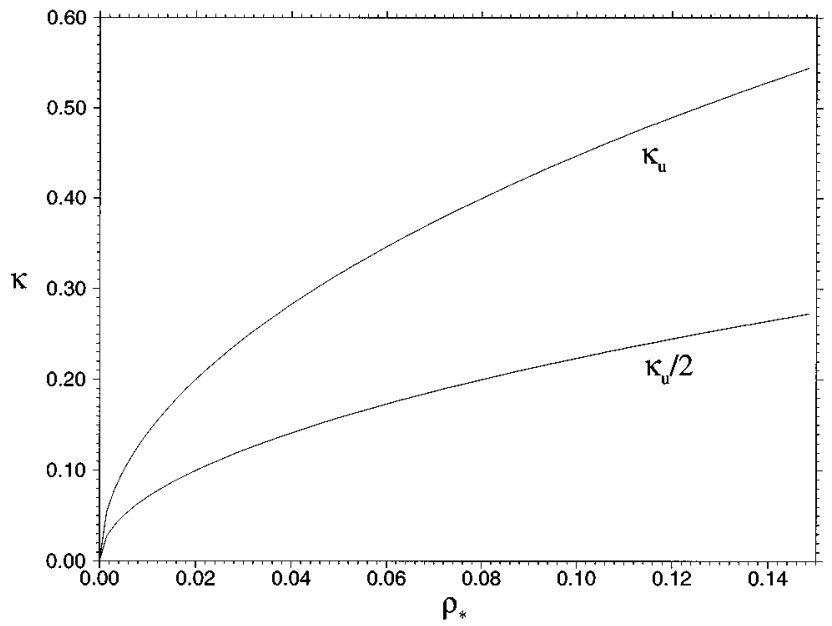

FIG. 1. Boundaries of the low-dimensional region.

$$
\partial_{x}^{2} n_{\mathrm{ens}}=-\partial_{x}^{2}\left|E_{\text {trunc }}(x, t)\right|^{2}
$$

we shall refer to this low-dimensional system as the $\rho$ triplet. In the general case where an arbitrary number of modes is allowed, it can be shown that if the density is related to the electric field as above, with the complete $E(x, t)$ field replacing $E_{\text {trunc }}(x, t)$, the resulting equation governing the Langmuir field turns out to be a nonlinear Schrödinger equation that is completely integrable; Eq. (6) indicates that such a kind of dynamics would take place only on the leftmost side of Fig. 1. In the narrow band given by Eq. (7) only lowdimensional dynamics can occur.

Let us then proceed to the study of the truncated system, postponing the appropriate numerical checkings for later on. On using expansion (8) with Eqs. (9) and (1) one readily obtains a Hamiltonian dynamical system with the phases $\phi$ as canonical coordinates and the amplitudes $\rho$ as their respective conjugated momenta. The Hamiltonian reads

$$
\begin{aligned}
H= & 2 \rho_{0} \sqrt{\rho_{+1} \rho_{-1}} \cos \psi-k^{2}\left(\rho_{+1}+\rho_{-1}\right)+\rho_{+1} \rho_{-1}+\rho_{+1} \rho_{0} \\
& +\rho_{-1} \rho_{0},
\end{aligned}
$$

with $\psi \equiv \phi_{+1}+\phi_{-1}-2 \phi_{0}$. Considering the form of the Hamiltonian, it is convenient to perform the following canonical transformation, which converts $\psi$ into a new canonical coordinate:

$$
\begin{gathered}
\phi_{+1}+\phi_{-1}-2 \phi_{0} \rightarrow \phi_{0}^{\prime}=\psi, \\
\rho_{0} \rightarrow-2 \rho_{0}^{\prime}, \\
\rho_{ \pm 1} \rightarrow \rho_{ \pm 1}^{\prime}+\rho_{0}^{\prime}, \\
\phi_{ \pm 1} \rightarrow \phi_{ \pm 1}^{\prime},
\end{gathered}
$$

and

$$
H \rightarrow H\left(\rho_{0}^{\prime}, \psi\right),
$$

where the primes denote new coordinates. The usefulness of the transformation is that it allows us to explicitly see the time conserved character of $\rho_{ \pm 1}^{\prime}$ since they appear as mo-

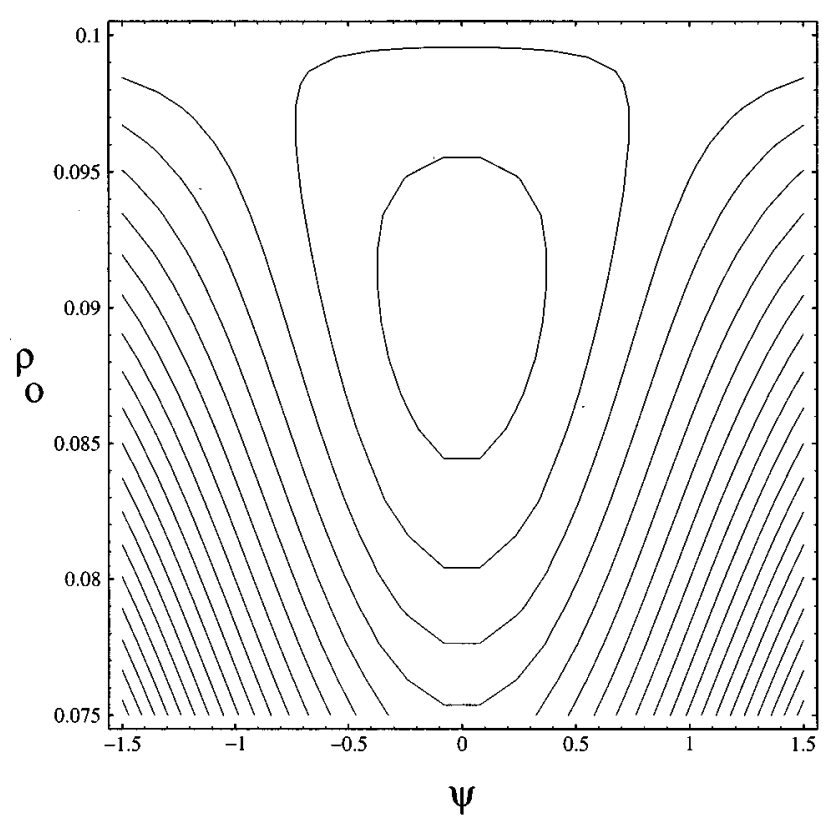

FIG. 2. Low-dimensional integrable trajectories on the $\left(\rho_{0}, \psi\right)$ plane for $\rho_{*}=0.1$.

menta conjugated to cyclic coordinates $\phi_{ \pm 1}^{\prime}$. At this point it seems appropriate to look at the dynamics generated by the Hamiltonian (10) and this is done with the contour plots of Fig. 2, where we record trajectories on $\left(\rho_{0}, \psi\right)$ space. To build up the contour plot we launch several initial conditions always keeping the same value for $\rho_{ \pm 1}^{\prime}$ with $\rho_{-1}^{\prime}=\rho_{+1}^{\prime}=\frac{1}{2} \rho_{*}, \rho_{*}=0.1$, and $k=0.9075 k_{u}$. There is no problem in taking $\rho_{ \pm 1}^{\prime}$ as a common value for various initial conditions at the present stage of the analysis, but one should verify whether this choice provides relevant information when we simulate the full system with its corresponding nonintegrable features. One could argue, for instance, why distinct values of $\rho_{ \pm 1}^{\prime}$ are not assigned to distinct initial conditions, exactly as done with regard to the energy (different orbits in a contour plot correspond to different values of $H$ ). Our point is that, so far, the transition to chaos basically involves only three active modes, with modes $m=+1$ and $m=-1$ sharing complete symmetry among themselves; the conserved quantity of the full system (1) and (2)

$$
\int_{0}^{L}|E(x, t)|^{2} d x=\text { const }
$$

guarantees that $\rho_{0}+2 \rho_{ \pm 1}=2 \rho_{ \pm 1}^{\prime}$ is a conserved quantity for the system irrespective of the complexity of the dynamics. Note that the when transition takes place one can no longer consider the ion-acoustic field as totally enslaved to the Langmuir field. This means that the approximation (9) and, subsequently, the constancy of $H$ break down. Equation (8), however, may still hold and this is the regime we are interested in.

From Fig. 2 one can still observe the positioning of the fixed points related to the $\rho$ triplet. The figure, which represents a typical orbital dynamics after the pitchfork bifurcation, reveals the presence of the fixed points mentioned ear- 


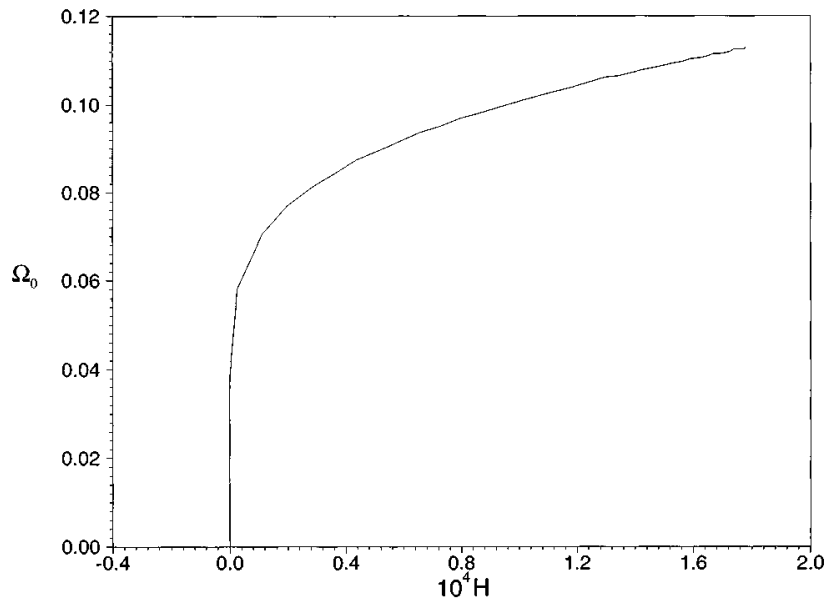

FIG. 3. Gyrofrequency $\Omega_{0}$ versus $H$ for $\rho_{*}=0.1$ and $k=0.9075 k_{u}$.

lier: two unstable points located at $\left(\rho_{0}=\rho_{*}=0.1, \psi \approx \pm 1\right)$ and one stable central fixed point (CFP) located at $\left(\rho_{0} \sim 0.09, \psi=0\right)$. Before the transition there is only one unstable fixed point at $\left(\rho_{0}>\rho_{*}, \psi=0\right)$ and any orbit launched with $\rho_{0}=\rho_{*}$ is stationary in the sense that $d \rho_{0} / d t=0$; see Ref. [6].

Another quantity of interest is the frequency with which trajectories circulate around this CFP. We refer to this frequency as $\Omega_{0}$, pointing out that it is a function of the numerical value of $H$ corresponding to the initial condition with which the orbit is launched. One can therefore write $\Omega_{0}=\Omega_{0}(H)$, noting that at the separatrix $H=0$. Referring to Fig. 2, we present a plot of the gyrofrequency $\Omega_{0}(H)$ versus $H$ in Fig. 3. From the figure one appreciates the usual behavior of frequency curves; the frequency reads a nonvanishing value at the CFP (rightmost extreme) whose absolute value we denote by $\Omega_{c}$, going to zero as one approaches the separatrix. This fact shall be seen to have relevant implications on the location of locked states.

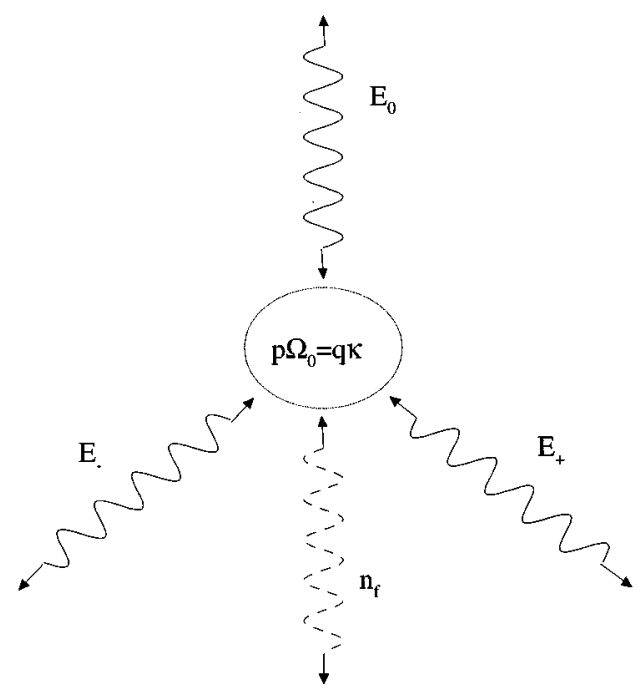

FIG. 4. Schematics of the interaction process involving the $\rho$ triplet and normal mode ion-acoustic fluctuations $n_{f}$.

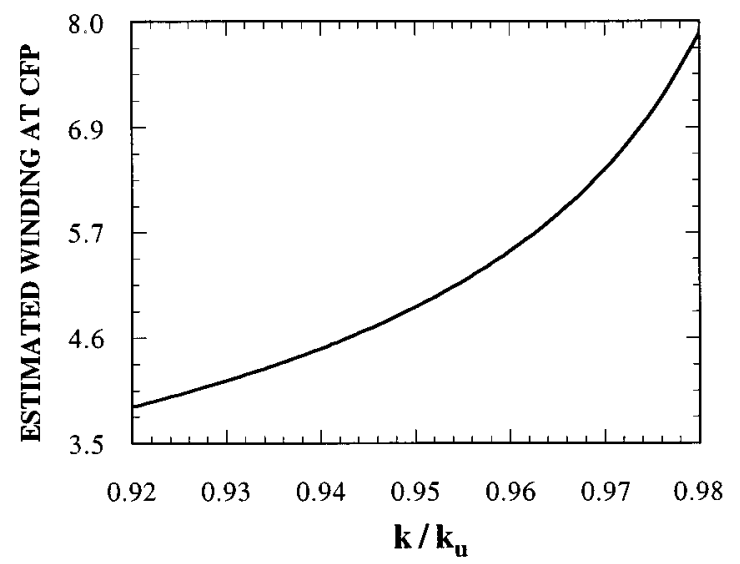

FIG. 5. Estimated winding at the CFP, $k / \Omega_{c}$, versus $k$ for $\rho_{*}=0.1$.

$\Omega_{c}$ can be calculated analytically [9]. In order to do so we expand the Hamiltonian $H\left(\rho_{0}^{\prime}, \psi\right)$ around the CFP. The expansion reads

$$
H\left(\rho_{0}^{\prime}, \psi\right) \approx \mathrm{const}+\frac{1}{2}\left[\left.\partial_{\rho_{0}^{\prime}}^{2} H\right|_{\mathrm{CFP}} \delta \rho_{0}^{\prime 2}+\left.\partial_{\psi}^{2} H\right|_{\mathrm{CFP}} \psi^{2}\right]
$$

and $\Omega_{c}$ is written as

$$
\Omega_{c}\left(k, \rho_{*}\right)=\sqrt{\left|\partial_{\rho_{0}^{\prime}}^{2} H \partial_{\psi}^{2} H\right|_{\mathrm{CFP}}}=\frac{2 \sqrt{\frac{2}{7}} k}{\sqrt{\frac{k^{2}}{\left(1-k^{2}\right)\left(\frac{3}{4}+k^{2}\right) k_{u}^{2}}}} .
$$

With the above tools one can now proceed to study the transition to chaos in the next section.

\section{TRANSITION TO CHAOS}

Now we perform a variety of simulations of Eqs. (1) and (2) to check our assumptions and to detect the transition to chaos. As it is somewhat usual for this kind of system, our simulation scheme consists of writing all the dynamical variables and the differential equations as Fourier series in the spatial variable. A number $N$ of modes ranging from $N=32$ to 128 for each dynamical variable is used, nonlinear products in the differential equations are evaluated with a fast Fourier transform (FFT) subroutine, and the set of temporal equations is advanced in time with a predictorcorrector algorithm. Both the FFT and the predictorcorrector algorithm are subroutines of a Cray Y-MP2E computer. Numerical precision is tested by requiring stability against variation of tolerance factors and by monitoring the time evolution of the conserved quantity

$$
\mathcal{H}=\int_{0}^{L}\left[\left|\partial_{x} E\right|^{2}+n|E|^{2}+\frac{1}{2}\left(n^{2}+v^{2}\right)\right] d x,
$$

with $\partial_{t} n=-\partial_{x} v$. In terms of $\mathcal{H}$, relative errors were found to be about one part in $10^{8}$.

Returning to physics, we have seen that if we operate in that region of Fig. 1 where the basic wave vector $k$ satisfies $k_{u}>k>k_{u} / 2$, the generation of harmonic modes with wave 

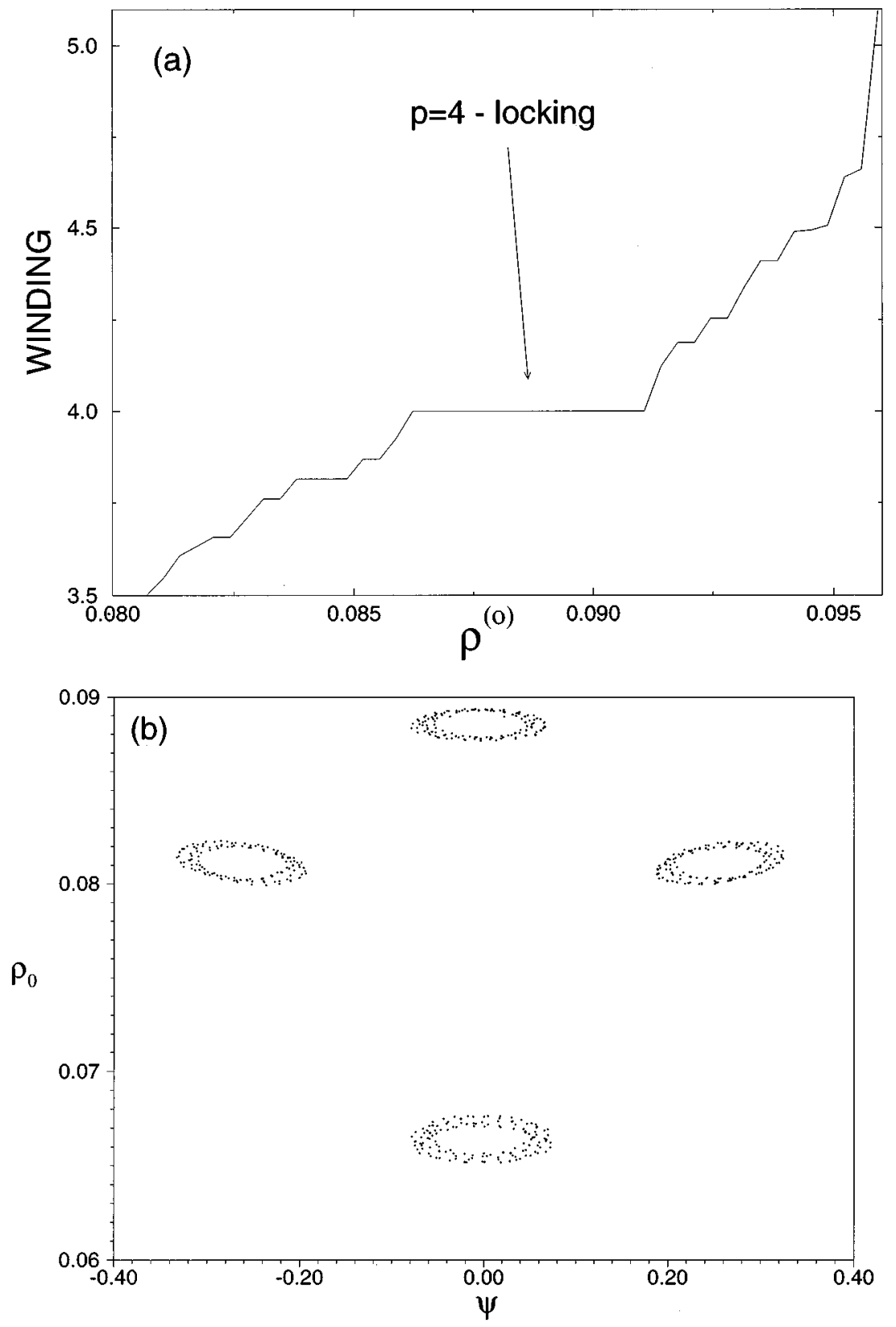

FIG. 6. (a) Winding number and $p=4$ locking: (b) Corresponding Poincaré plot and resonant island. $\rho_{*}=0.1, \quad k=0.9075 k_{u}$, $t_{\text {final }}=4644.51$, and there are approximately 300 ion-acoustic cycles. vectors $\pm 2 k, \pm 3 k, \pm 4 k, \ldots$ is greatly inhibited: this will be confirmed with the coming numerical simulations. In addition, if one also satisfies either condition (6) or (7), ionacoustic modes are enslaved to the truncated Langmuir field through relation (9). In the first case where ion-acoustic modes are enslaved regardless of the value of $k$, the transition to chaos is unlikely because the system can be always approximated by an integrable model governed by the Hamiltonian (10). In the second case where the magnitude of $\rho_{*}$ is not too small, the situation changes. The ion-acoustic field will be enslaved only if one is really close to $k_{u}$. As soon as that region is abandoned, nonintegrable features take place, driving the transition. The question is what kind of nonintegrable features are likely to be expected. In order to address the issue, we proceed as follows. We decompose the density associated with the ion-acoustic field in the form

$$
n(x, t)=n_{\mathrm{ens}}(x, t)+n_{f}(x, t) .
$$

In that equation $n_{f}$ accounts for the free $(f)$ component of the total density, i.e., the component not enslaved to the Langmuir field under the static approximation. We expect this component to be the responsible for the transition since $n_{\text {ens }}$ alone generates only regular dynamics.

The dynamical equation governing the basic Fourier component of $n_{f}$, for instance, can be written in the form

$$
\left(d_{t}^{2}+k^{2}\right) n_{f, 1}(k, t)=k^{2}\left(\frac{d_{t}^{2}\left(|E|^{2}\right)_{1}(k, t)}{k^{2}}\right),
$$

with $d_{t} \equiv d / d t$. The right-hand side term, the one containing the electric field, can thus be seen as a source driving $n_{f}$ oscillations. Once again one observes that if $d_{t}^{2} \ll k^{2}$ the source term is small and $n_{f} \rightarrow 0$. On the other hand, as soon as the nonlinear frequency of the $\rho$ triplet becomes comparable to $k$, Eq. (15) suggests that $n_{f}$ can no longer be dis- 


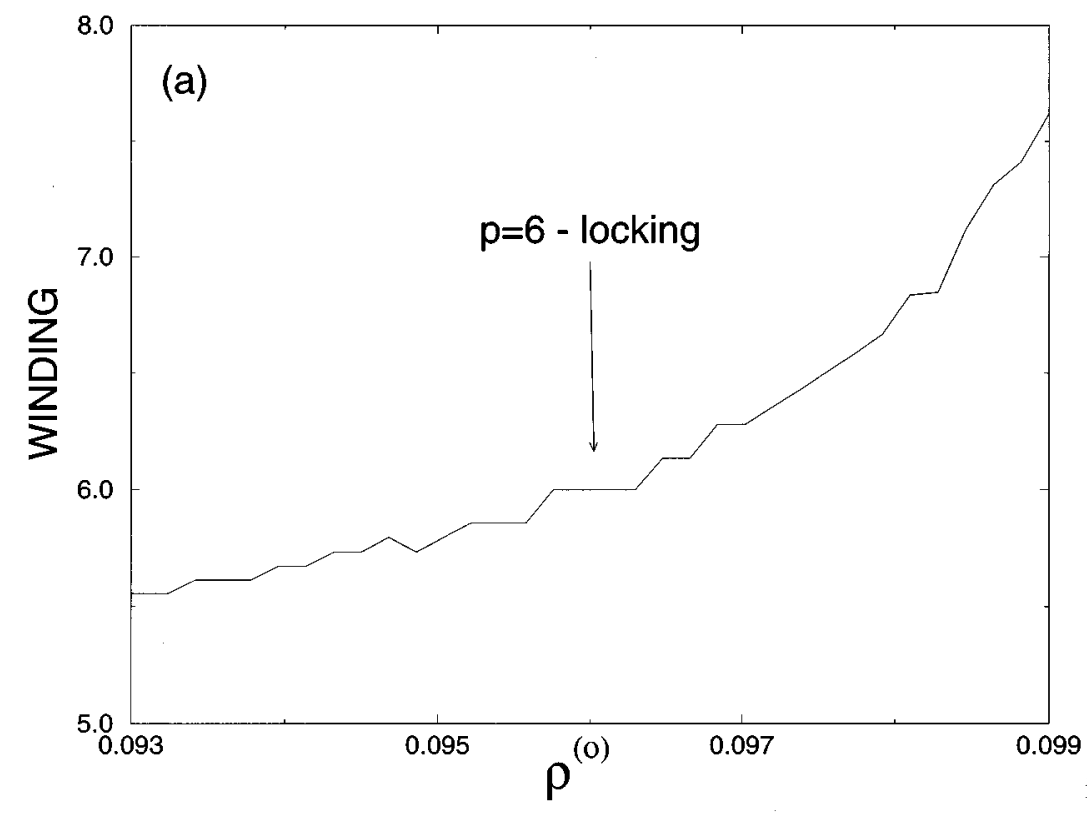

FIG. 7. (a) Winding number and $p=6$ locking: (b) Corresponding Poincare plot and resonant island. $\rho_{*}=0.1, \quad k=0.9425 k_{u}$,

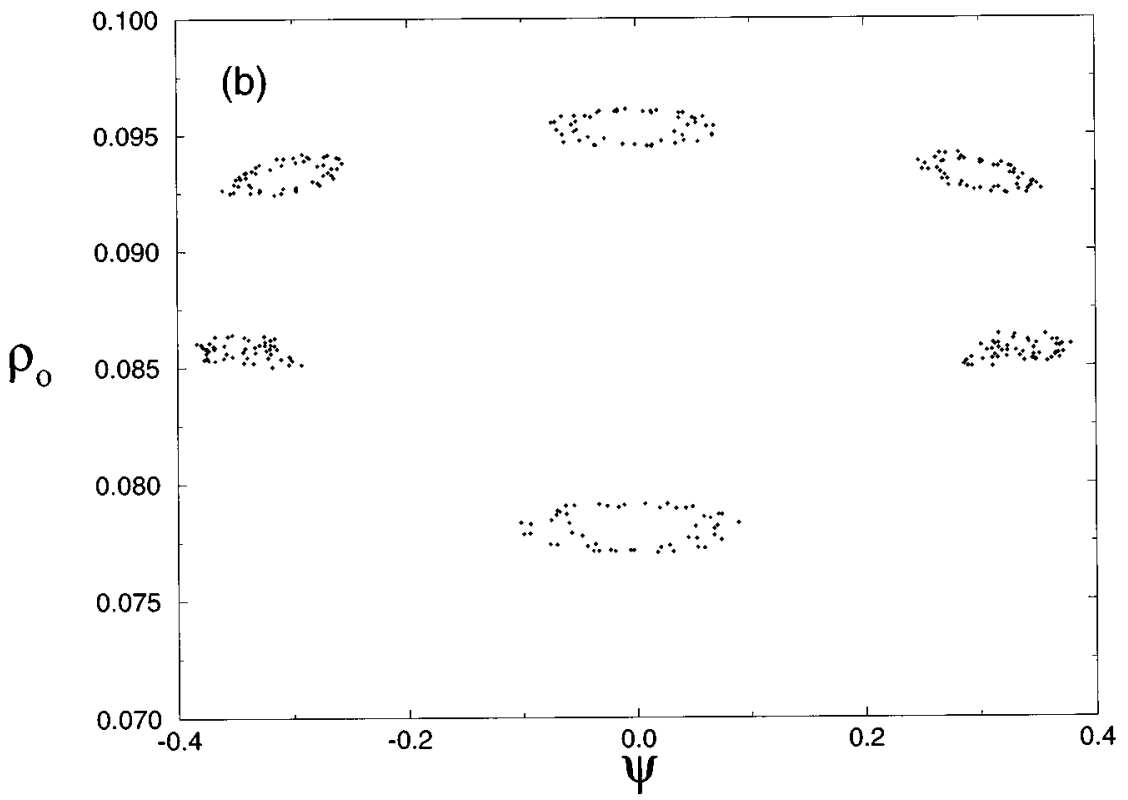
$t_{\text {final }}=4472.03$; there are approximately 300 ionacoustic cycles.

carded; this is the situation where one should look for chaos. Equivalent equations can be written down for higher Fourier components and similar reasoning is applicable. In any case, numerical simulations will reveal that the role played by the $n_{f}$ at the fundamental wave vector $k$ is the most relevant.

An examination of Eq. (15) in conjunction with the equation for the slow amplitude of the electric field, Eq. (1), also suggests that transition to chaos is marked by nonlinear locking or nonlinear resonance. Indeed, due to the form of the nonlinearity one is lead to think that resonances are present and that in order to see one of them one should meet the condition

$$
p \Omega_{0} \approx q k
$$

with $p$ and $q$ integers, since $k$, as we recall, is approximately the oscillatory frequency of $n_{f}$ and $\Omega_{0}$ is the nonlinear fre- quency of the $\rho$ triplet. The schematics for the nonlinear interaction is displayed in Fig. 4.

The relation (16) indicates how nonlinear resonances are to be located. Accordingly, what we actually do here goes as follows. We first plot the estimated winding number at the CFP $k / \Omega_{c}$ as a function of $k$ keeping $\rho_{*}$ fixed. We choose $\rho_{*}=0.1$ and $q=1$ throughout the paper, displaying the plot in Fig. 5. The condition $q=1$, in particular, enables one to work with stronger resonances, which can be more easily seen on phase space. With the plot we estimate the particular value of $k, k_{p}$, where a $p$ resonance or $p$ locking is born: it suffices to evaluate it from $k_{p} / \Omega_{c}\left(k_{p}\right)=p$, where $p$ is the integer naming the resonance. Now, by virtue of the shape of the gyrofrequency curve represented in Fig. 3, for slightly smaller values of $k$ the resonance moves away from the CFP towards the separatrix. In particular, the conditions for observing the resonance become ideal when it is not too close to either the CFP or to the separatrix. 


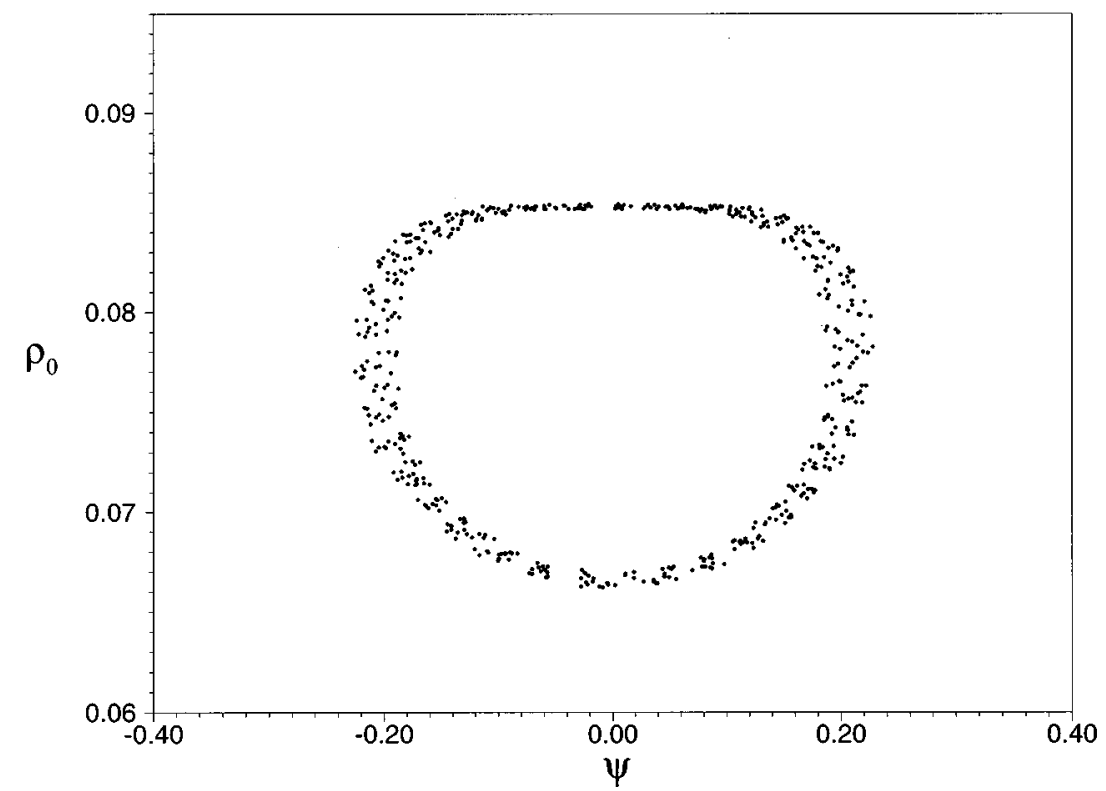

FIG. 8. Quasiperiodic cycle for the same parameters used in Fig. 6(b).

To actually see the locking we proceed to simulations in two steps. First of all we plot the simulated winding number against $\rho_{0}(t=0) \equiv \rho^{(0)}$ considering $\phi_{0}(t=0)=0$, to precisely locate the resonance. The winding $\mathcal{W}$ here is used in the form [10]

$$
\mathcal{W} \equiv \lim _{t \rightarrow \infty}\left[\frac{N_{n_{f}}}{N_{\rho_{0}}}\right],
$$

the capital $N$ representing the number of cycles of the respective variables appearing as subscripts. If there is locking involving the $\rho$ triplet and $n_{f}$, a plateau should appear in the figure. From Fig. 5, in the case $p=4$ locking is expected for $k<k_{4} \approx 0.92 k_{u}$ because for larger values of $k$, the resonance is not yet born. We take $k=0.9075 k_{u}$ into the simulations to produce Fig. 6(a). The plateau is clearly devised for which $p=4$. The second step is to ask how the locked state manifests itself in a Poincare plot constructed with the coordinates $\rho_{0}$ and $\psi$ of the unperturbed $\rho$ triplet; compare with Fig. 2. The answer is given in Fig. 6(b), where we plot the pair $\left(\rho_{0}, \psi\right)$ each time $\operatorname{Re}\left\{n_{f}(k, t)\right\}$ attains a maximum; $d_{t} \operatorname{Re}\left\{n_{f}(k, t)\right\}=0, d_{t}^{2} \operatorname{Re}\left\{n_{f}(k, t)\right\}<0$. The simulation runs until a final time $t_{\text {final }}=4644.51$, corresponding approximately to 300 ion-acoustic cycles, and a chain of four resonant islands is seen encircling the CFP, as expected. In Fig. 7 we investigate the behavior of $\Omega_{c}$ in another range of values of $k$ such that a $p=6$ locking would be present; this will be useful in what follows. It is seen from Fig. 5 that if $k<k_{6} \approx 0.95 k_{u}$ then one could expect to see the $p=6$ locking. In Fig. 7(a) the winding is shown for $k=0.9425 k_{u}$, actually displaying the $p=6$ locking, and in Fig. 7(b) the corresponding resonant chain is seen; simulations run until $t_{\text {final }}=4472.03$ and the number of ion-acoustic cycles is again

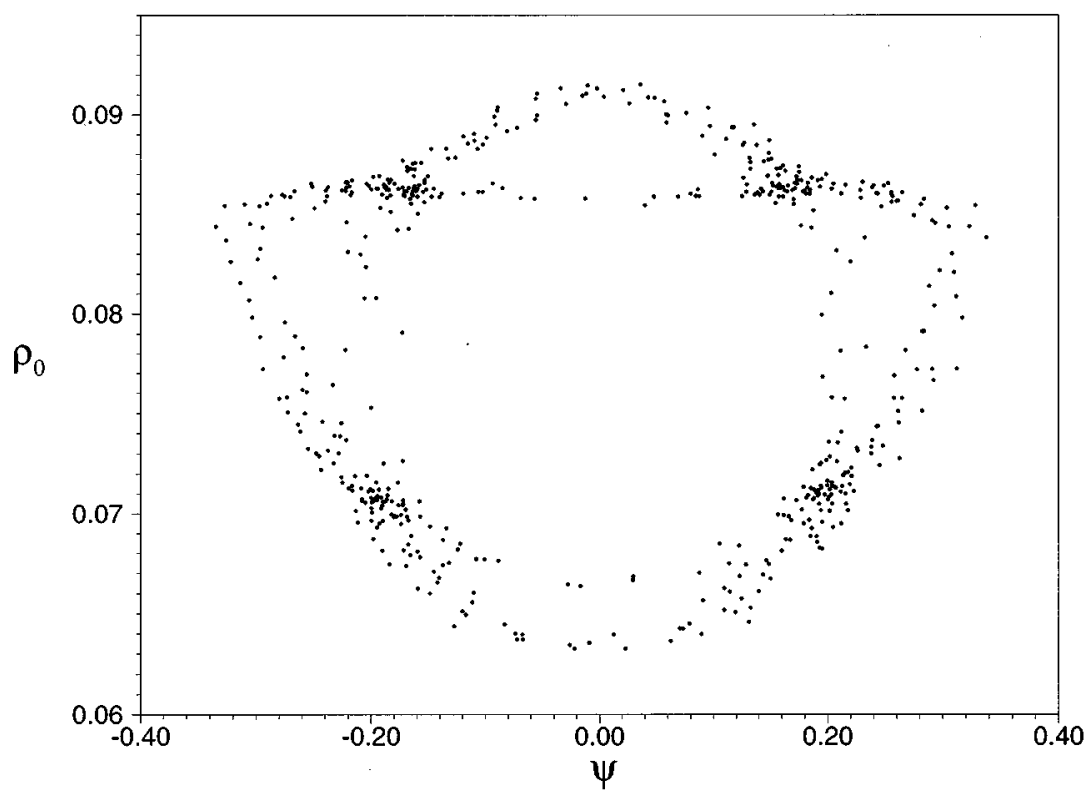

FIG. 9. Separatrix of the $p=4$ resonant island. 



FIG. 10. Power spectra of the $p=4$ locking: (a) corresponding to the situation of Fig. 6(b) and (b) corresponding to the situation of Fig. 9. approximately 300. For sake of comparison, in Fig. 8 we use the same parameters as in Fig. 6(b) to make a Poincaré plot of an initial condition corresponding to the absence of locking: we choose an initial condition off the plateau. In this case the figure reveals the familiar quasiperiodic behavior, where a full curve replaces the discrete islands.

Besides locking, another important feature of a resonant island is its ability in developing chaotic activity near its separatrix. It is known indeed that chaos first appears around the separatrix as a result of homoclinic and heteroclinic crossings. Let us make sure that in our case chaos is actually stronger on that location. To see that, let us first analyze the phase space in order to pinpoint a separatrix. This is done in the Poincare plot of Fig. 9, where we consider the same parameters as in Fig. 6(b), with the exception of $\rho^{(0)}$, which is slightly displaced relatively to the previous value; this new value of $\rho^{(0)}$ lies closer to the leftmost side of the plateau of
Fig. 6(a). Figure 9 suggests that the final effect of the displacement is actually bringing the system nearer the separatrix: at least the orbit represented in the figure looks like a separatrix orbit. Now we return to the original question: is chaos stronger if one is closer to this apparent separatrix? One single way to look at the issue is to perform a power spectrum analysis of the orbit. This is done in Fig. 10. In Fig. 10(a) we plot the power spectrum of $\rho_{0}(t)$ relative to the situation depicted in Fig. 6(b) and in Fig. 10(b), relative to the situation depicted in Fig. 9. In the first case, the one farther from the separatrix, the spectral distribution contains several spikes, which suggests subharmonic generation but not chaos. As for the second case, the distribution is spread out, which reveals the presence of stronger chaotic activity. The conclusion is that the first manifestations of chaos in our system are really connected to nonlinear locked states and the corresponding nonlinear resonances. 


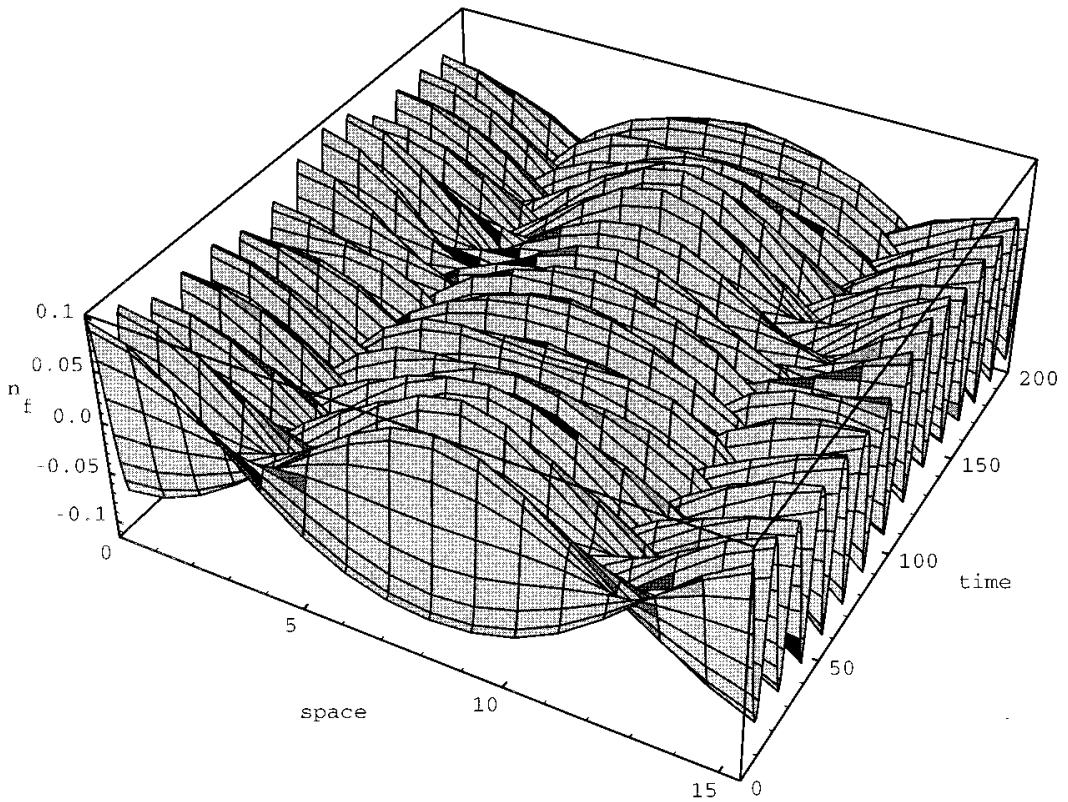

FIG. 11. Space-time history of $n_{f}(x, t)$. Parameters and initial condition are the same as those used in Fig. 6(b).

\section{COMPARISON WITH A LOW-DIMENSIONAL NONINTEGRABLE MODEL AND ASYMPTOTIC STATES OF THE FULL SYSTEM}

Figure 11 shows the space-time history of $n_{f}(x, t)$ for the same parameters and initial conditions used in Fig. 6(b). From the figure it becomes apparent that the free density fluctuations follow a quasistationary pattern with amplitude approximately constant and characteristic period $T_{f} \sim 14$, as it should be if one estimates the period from $T_{f} \sim 2 \pi / k$ with $k=0.9075 k_{u}$, which yields $T_{f} \sim 15$. This pattern is coherent with the previsions of Ref. [8]. In this reference it is suggested that nonintegrablity in this kind of system requires two counterpropagating ion-acoustic waves such that the second-order differential operator in Eq. (2) cannot be lowered to a first-order operator. If one had a single free- streaming ion-acoustic wave, the order could be reduced via use of characteristic coordinates.

These observations help us to construct a very simple low-dimensional nonintegrable model for our system, which keeps the basic characteristics observed in the simulations. To construct the nonintegrable model we simply replace $n_{\text {ens }}$ in our integrable model by $n_{\text {ens }}+n_{f}$, where $n_{f}$ is written as $n_{f}=N_{0} \cos (k t) \cos (k x)$. The amplitude $N_{0}$ is determined from direct inspection of the simulations: in general the amplitude is proportional to $\rho_{*}$ whose magnitude is, in turn, proportional to the driving term in Eq. (15).

Bearing all these facts in mind, we now present a Poincaré plot of the low-dimensional nonintegrable model in Fig. 12 using $\rho_{*}=0.1, k=0.9075 k_{u}$, and $N_{0}=0.1$. The final result is quite similar to the one depicted in Fig. 6(b). The

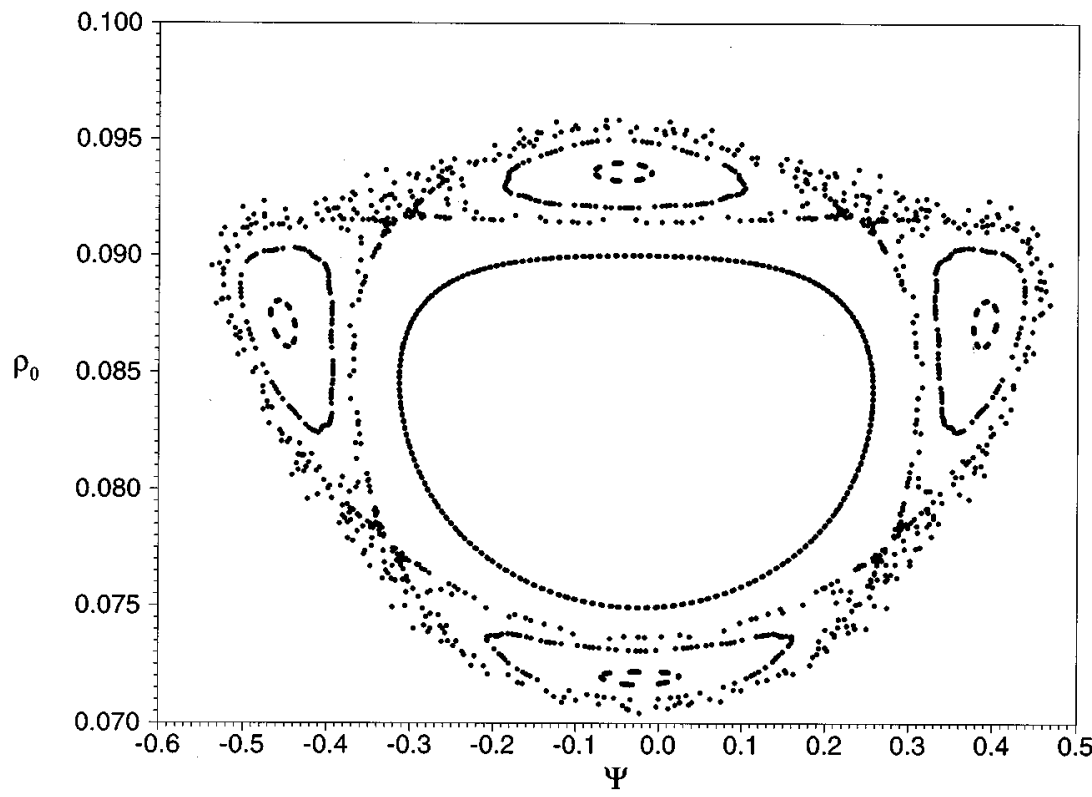

FIG. 12. Poincare plot of the low-dimensional nonintegrable model. Same parameters as in Fig. $6(b)$. 

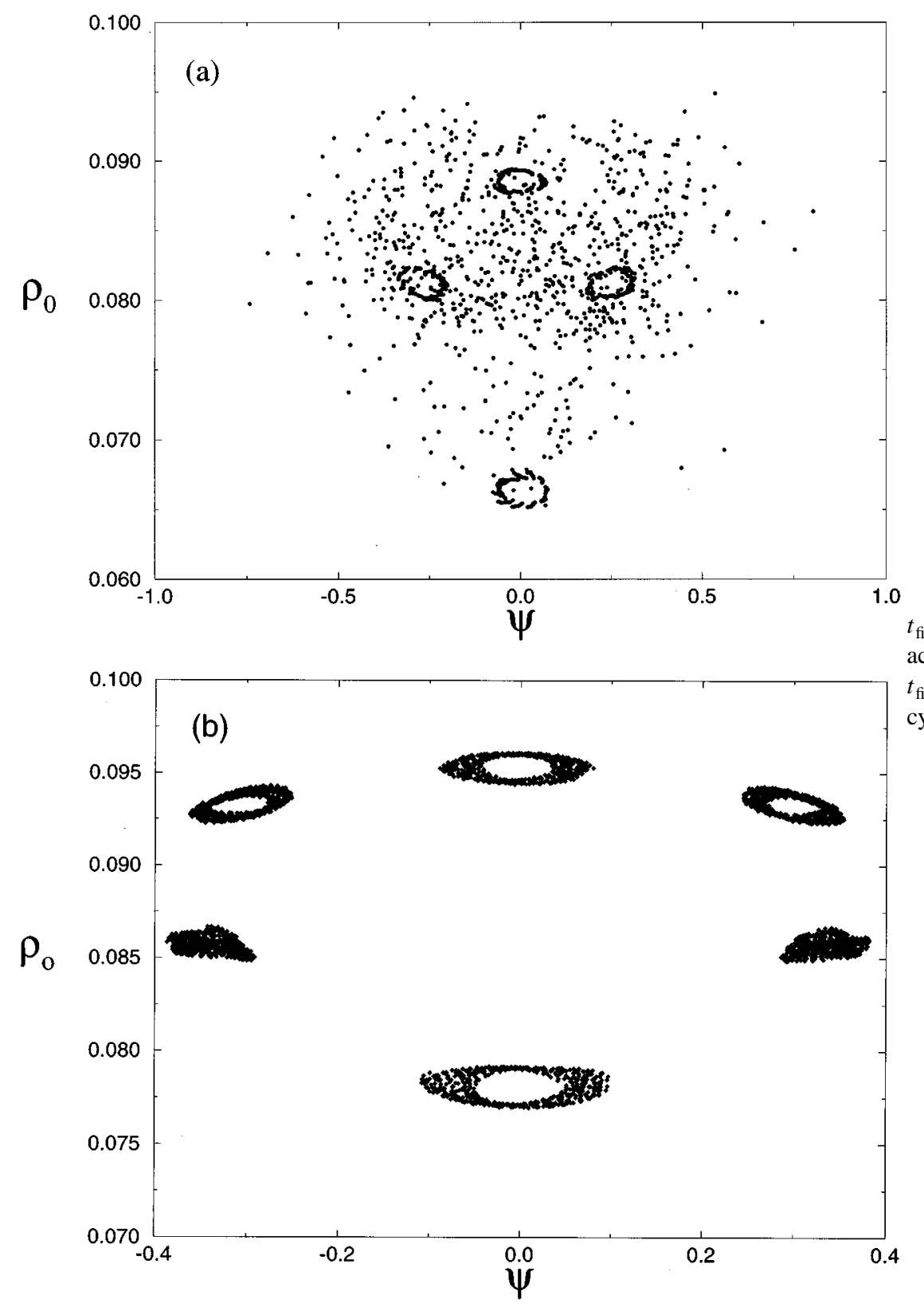

FIG. 13. Long time runs for (a) $p=4$ locking, $t_{\text {final }}=11611.3$, and approximately 750 ionacoustic cycles and (b) $p=6$ locking, $t_{\text {final }}=74533.8$; approximately 5000 ion-acoustic cycles.

similarity appears to indicate that the overall dynamics of the full system can be completely understood in terms of the nonintegrable model. However, we shall see next that the situation is a little more delicate.

The low-dimensional nonintegrable model gives birth to stable resonant chains. However, as one goes back to the full simulation and lets the simulation time increase, we see that sometimes the chains may not be the final asymptotic state of the interaction. This is shown in Fig. 13(a), where the simulation performed with the same parameters as in Fig. 6(b) runs until the final time $t_{\text {final }}=11611.3$, which corresponds approximately to 750 ion-acoustic cycles. Clear detuning, or diffusion, on phase space is seen as opposed to the previous shorter run where the number of cycles was about 300 . We would like to know what drives the detuning and the subsequent diffusion. As it appears, detuning seems to arise as a result of coupling of the $\rho$ triplet with high-frequency modes characterized by wave vectors $2 k$ or larger. This conclusion is immediate because if one suppresses artificially these higher-harmonic modes, similarly as is done in our lowdimensional nonintegrable model, detuning does not take place during arbitrarily long simulation intervals and the asymptotic state turns out to be the $p=4$ locking. An equivalent but realistic way to look at this feature without any artificial suppression of active modes is the following. Consider a resonance that occurs for values of $k$ closer to the instability threshold $k_{u}$ than the values we have been working with up until now. If such is the case, higher harmonics $m k$, with $|m|=2,3, \ldots$, would be as far as possible from the instability band and their participation in the interaction would be greatly reduced. Under such conditions one could also expect a behavior of the full system closely resembling that of the low-dimensional nonintegrable system. In particular, the resonance island would be likely to become a very stable entity of the system, in contrast to the transient aspect of the $p=4$ locking seen earlier. To investigate this possibil- 
ity, in Fig. 13(b) we use the same parameters used in Fig. 7 (b) to plot the $p=6$ island, with the exception of a longer simulation time $t_{\text {final }}=74533.8$ corresponding to 5000 ionacoustic cycles. Recalling that the wave vector here is closer to $k_{u}$, the stability of the resonance shown in the figure really appears to inform that the closer $k$ is to $k_{u}$, the smaller the participation of higher harmonics is and the more stable the low-dimensional locked states are.

The $p=6$ locking survives even if the simulation runs for as large as 10000 ion-acoustic cycles. We could not determine whether a threshold exists such that if $k$ is above it, but below $k_{u}$, locking is the asymptotic state, or if the detuning effect is so weak that diffusion does not manifest itself within the time intervals used in the simulations. In any case, for practical purposes it seems that if one is sufficiently close to $k_{u}$, but of course not so close as to make $n_{f} \rightarrow 0$ and the system integrable, locking can be seen as a stable and robust structure.

The importance of this feature is that low-dimensional locked states, under the weak instability condition $k \rightarrow k_{u}$, can be seen as asymptotic states to which initial disturbances tend and stay in a stable fashion after some small transient during which the ion-acoustic field becomes enslaved to the high-frequency electric field. On the other hand, as $k$ begins to move away from $k_{u}$ it has been seen that the stability of the locked state weakens in the sense that the resonant state tends to blur after long but finite time scales. These features are somewhat similar to what has been found in Ref. [11], where nonlinear coupling between a low-dimensional nonintegrable system and higher harmonics induces Arnol'd diffusion by means of which energy flows from small to large wave vectors. In our particular case, one would be tempted to say that stability of locking causes energy to be confined within the low-dimensional nonintegrable model, inhibiting all the dynamics related to very small spatial scales, such as collapse, cavitation, and Landau damping. Previous simulations point in that direction. To close this section, we mention that the higher chaotic activity near the separatrix is independent of locking stability as long as locking exists for a large number of ion-acoustic cycles.

\section{FINAL REMARKS}

In this paper we have investigated the transition to chaos in the Zakharov equations. The kind of approach we have adopted is similar to the one used in some very recent papers where a periodic and dissipationless model is used, which displays the presence of few unstable modes [4]. We have detected that the transition involves the presence of nonlinear locked states preceding chaos. We find that these states appear when the parameters governing the system are such that one is close to a pitchfork bifurcation destabilizing an homogeneous equilibrium. Under such condition one fulfills the requirement of a small number of active modes and locked states are likely. We have numerically observed these states that mark transition to chaos. A low-dimensional model is presented that contains the basic characteristics of locking.

Then, as one moves away from the pitchfork bifurcation, it was observed that the locked states lose their stability. What happens then is that if one is not sufficiently close to the bifurcation point, higher harmonics of the high-frequency field cannot be discarded and locking is perturbed, taking place only during finite, although very long, time intervals. On the other hand, if one is close enough to the bifurcation point, locking appears to survive over arbitrarily large periods of time. Some kind of sharp change as $k$ is varied may exist that divides the asymptotic behavior of the system into locked and unlocked final states.

In any case, chaos has been found to be stronger if one is close to the separatrix associated with the related resonant island. This suggests that locked states signal the start of the transition to chaos, just like what happens in lowdimensional systems.

\section{ACKNOWLEDGMENTS}

The authors wish to thank Iberê L. Caldas for helpful and interesting discussions. This work was partially supported by Financiadora de Estudos e Projetos (FINEP) and Conselho Nacional de Desenvolvimento Científico e Tecnológico $(\mathrm{CNPq})$, Brazil. Numerical computing was performed on the CRAY Y-MP2E at the Universidade Federal do Rio Grande do Sul Supercomputing Center.
[1] S.G. Thornhill and D. ter Haar, Phys. Rep. Phys. Lett. C 43, 43 (1978).

[2] P.A. Robinson and D.L. Newman, Phys. Fluids B 1, 2319 (1989).

[3] H.T. Moon, Phys. Rev. Lett. 64, 412 (1990).

[4] X.T. He and C.Y. Zheng, Phys. Rev. Lett. 74, 78 (1995).

[5] S.E. Gibson, D.L. Newman, and M.V. Goldman, Phys. Rev. E 52, 558 (1995).

[6] G.I. de Oliveira, F.B. Rizzato, and A.C.-L. Chian, Phys. Rev. E 52, 2025 (1995).
[7] A.C.-L. Chian and C.F. Kennel, Astrophys. Space Sci. 97, 9 (1983).

[8] N. Yajima and M. Oikawa, Prog. Theor. Phys. 56, 1719 (1976).

[9] A.J. Lichtenberg and M.A. Lieberman, Regular and Stochastic Motion (Springer, New York, 1983).

[10] G. Corso and F.B. Rizzato, Physica D 80, 296 (1995).

[11] J. DeLuca, A.J. Lichtenberg, and S. Ruffo, Phys. Rev. E 51, 2877 (1995). 